\title{
Simultaneous enhancement of cellular and humoral immunity by the high salt formulation of $\mathrm{Al}(\mathrm{OH})_{3}$ adjuvant
}

\author{
Cell Research (2017) 27:586-589. doi:10.1038/cr.2017.14; published online 20 January 2017
}

\section{Dear Editor,}

Adjuvants play essential roles in vaccination by enhancing and/or shaping antigen-specific immune responses [1]. They are commonly used in every type of vaccines, e.g., cancer vaccines, which have been proved to be a promising approach for cancer immune therapy [2]. Aluminum-based adjuvants have been widely used in human vaccination for decades [3]. Despite the robust induction of antibody-mediated immune responses, a limitation of aluminum-based adjuvants is their weak stimulation of cell-mediated immunity [4]. Thus, the development of safe, stable and effective adjuvants with the ability to enhance both humoral and cellular immune responses during clinical vaccination remains challenging. As the major antigen-presenting cells (APCs) to initiate immune responses, dendritic cells (DCs) can be triggered by environmental stimulation to elicit different phenotypes and functions [5, 6]. Moreover, cross-presentation of extracellular protein antigens by DCs contributes to the generation of cytotoxic T lymphocyte (CTL) responses [7]. Sodium chloride $(\mathrm{NaCl})$ is closely related to our daily lives and has recently been found to have potential effects on the development of autoimmune diseases [8, $9]$ and specific states of macrophages [10]. However, the impacts of high concentrations of salt on the functions of DCs and the immune system have not been studied. The use of high salt formulation in combination with aluminum adjuvants in protein vaccines remains to be explored.

Here we developed a new formulation of aluminum hydroxide $\left(\mathrm{Al}(\mathrm{OH})_{3}\right)$ adjuvant with a high salt concentration and utilized the OVA model antigen and the HBsAg antigen to evaluate its adjuvant effect. The serum ELISA results showed that the increase of the total $\mathrm{IgG}$ level reached a peak at $3.6 \% \mathrm{NaCl}$ (Figure 1A). Compared with the adjuvant prepared in regular $\mathrm{NaCl}$ concentration $(0.9 \%)$, the high-salt formulation of $\mathrm{Al}(\mathrm{OH})_{3}$ mildly improved OVA-induced production of IgG1-associated Th2 responses rather than IgG2a- and IgG2b-associated Th1 responses (Figure 1A). Similar results were also observed in the HBsAg model (Supplementary information, Figure S1A). These data suggest that the high-salt formulation of $\mathrm{Al}(\mathrm{OH})_{3}$ enhances humoral immunity by stimulating the $\mathrm{Th} 2$ response.

Next, we assessed whether the new formulation can enhance the induction of cell-mediated immunity. We found that vaccination of mice with $\mathrm{OVA} / \mathrm{Al} / 3.6 \% \mathrm{NaCl}$ dramatically increased the frequency of OVA-specific $\mathrm{CD} 8^{+} \mathrm{T}$ cells as detected by PE-conjugated $\mathrm{OVA}_{257-264^{-}}$ bound $\mathrm{H}-2 \mathrm{k}^{\mathrm{b}}$ tetramer compared with vaccination with OVA/Al (Figure 1B). In addition, vaccination with OVA $/ \mathrm{Al} / 3.6 \% \mathrm{NaCl}$ not only dramatically increased the frequency of $\mathrm{CD} 8^{+} \mathrm{INF}-\gamma$-producing CTLs, but also increased the secretion of INF- $\gamma$ (Figure 1C and 1D). Similar results were also obtained in the HBsAg vaccine model (Supplementary information, Figure S1B and S1C). Moreover, $\mathrm{T}$ lymphocytes derived from the mice immunized with OVA/ $\mathrm{Al} / 3.6 \% \mathrm{NaCl}$ exhibited higher cytotoxicity against E.G7-OVA cells (a cell line expressing OVA peptides) as revealed by a standard ${ }^{51} \mathrm{Cr}$-release assay (Figure 1E). Notably, there was no significant increase in the percentages of $\mathrm{CD}^{+} \mathrm{INF}-\gamma$-producing Th1 cells after high salt treatment in either the OVA or HBsAg vaccine model (Supplementary information, Figure S1D), indicating that high salt helped to enhance the induction of antigen-specific CTLs, bypassing the $\mathrm{CD}^{+} \mathrm{T}$ helper arm. Also, there was no increase of the IL-4 level after immunization with $\mathrm{OVA} / \mathrm{Al} / 3.6 \% \mathrm{NaCl}$ compared with the OVA/Al group (Supplementary information, Figure S1E).

We next examined whether and how high salt influences the activation of DCs. Since a $\mathrm{NaCl}$ concentration of $1.8 \%$ caused increased apoptosis and cell death of DCs (Supplementary information, Figure S1J), our subsequent in vitro experiments mainly focused on the concentrations of $1.2 \%$ and $1.5 \%$. Interestingly, high salt significantly promoted maturation and antigen uptake ability of DCs in vitro (Figure $1 \mathrm{~F}$ and $1 \mathrm{G}$ and Supplementary information, Figure S1F). Moreover, high salt promotes the expression of inflammatory cytokines in DCs at both mRNA and protein levels (Supplementary 
A
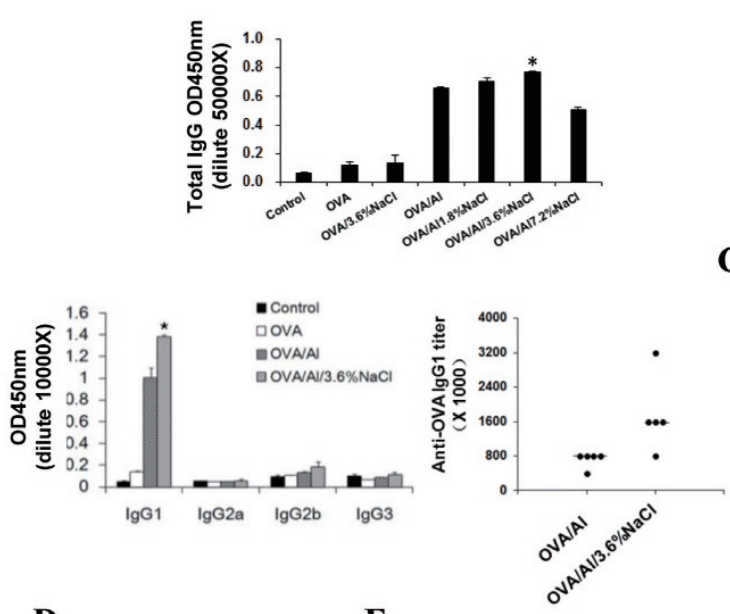

D

E

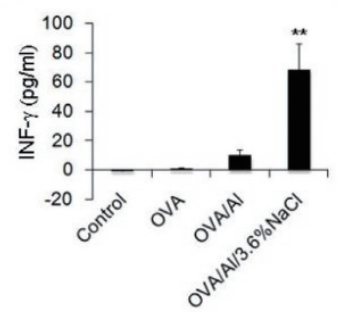

G
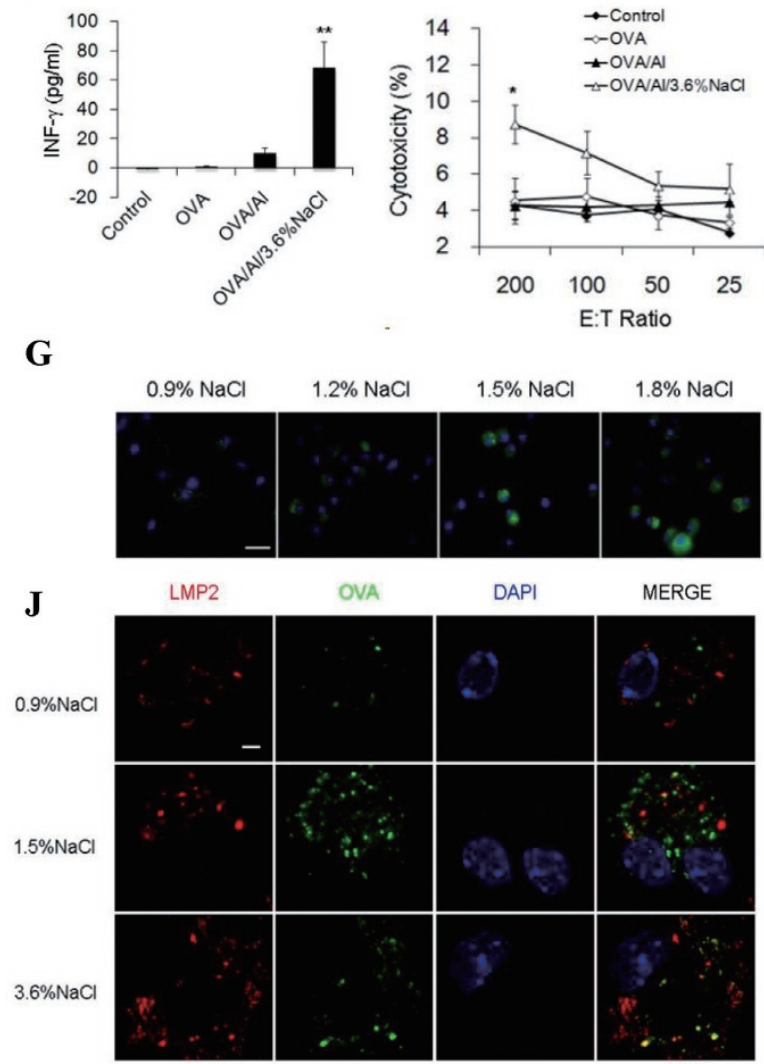

$\mathbf{K}$

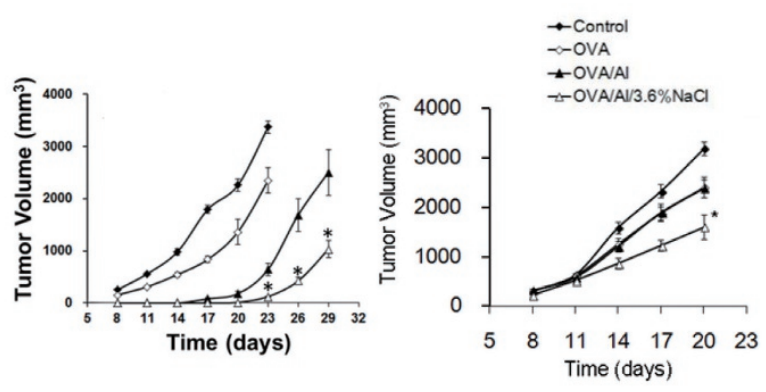

B

C

H

I

$\mathbf{L}$
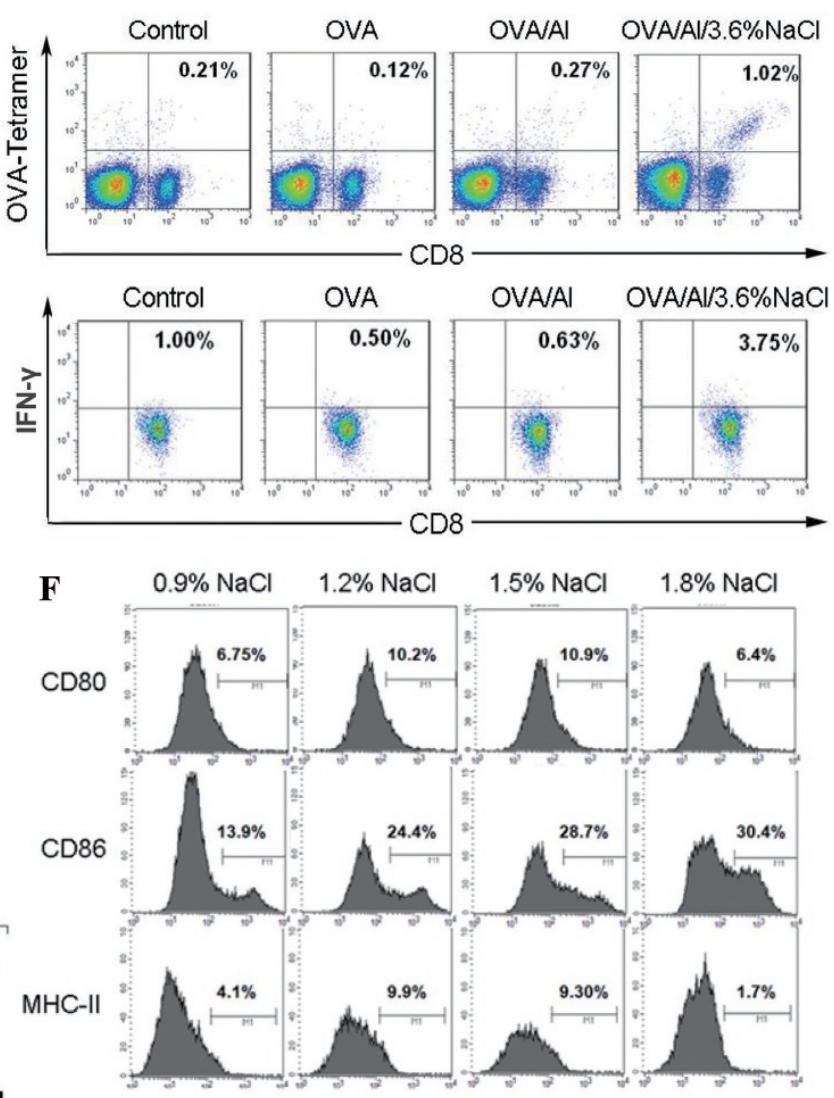

H
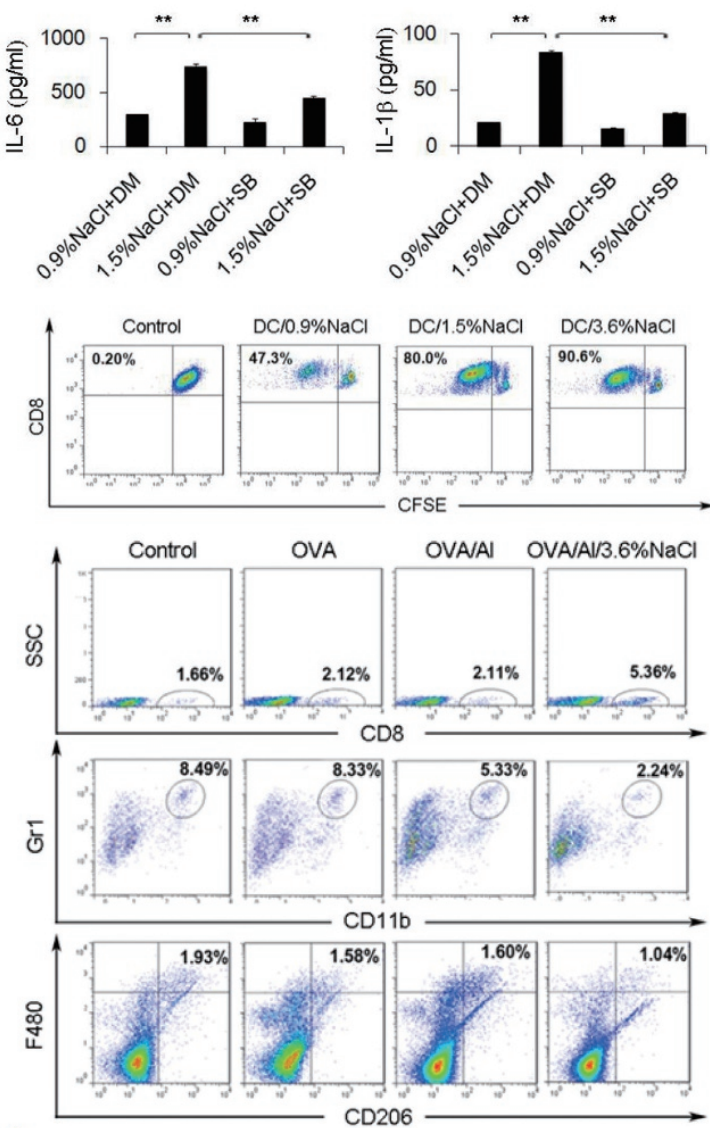
information, Figure S1G and S1H). As MAPK pathways were reported to have roles in cellular responses to stress stimulation [11], we explored the possibility of the involvement of the MAPK signaling pathway in DC activation. The phosphorylation of p38 MAPK significantly increased after hours of high salt treatment (Supplementary information, Figure S1I). Furthermore, the increased release of inflammatory cytokines could be abolished by the addition of a p38 inhibitor SB203580 (Figure 1H).

Efficient cross-presentation of extracellular proteins by DCs has an important role in the initiation of immune responses [7]. Here, we used $\mathrm{CD} 8^{+} \mathrm{T}$ cells from OT-I mice (specific for the $\mathrm{H}-2 \mathrm{~K}^{\mathrm{b}} / \mathrm{OVA}$ complex) to test whether high salt-stimulated DCs can perform cross-presentation of soluble OVA. The FCM analysis of CFSE fluorescence showed a 2-fold increase in $\mathrm{CD}^{+} \mathrm{T}$-cell proliferation upon co-culture with high salt-treated DCs (Figure 1I). Also, more clusters of DCs and $\mathrm{CD} 8^{+} \mathrm{T}$ cells were observed and enhanced secretion of IL-2 was detected in the supernatants during the co-culture process (Supplementary information, Figure S1K and S1L). Moreover, confocal microscopy revealed that more OVA co-localized with LMP2 (a marker of the immunoproteasome) in high salt-treated DCs, suggesting a more effective processing of antigens (Figure 1J). Collectively, the above data indicate that high salt can significantly enhance cross-presentation of extracellular antigens by DCs, which might play a critical role in the induction of antigen-specific CTL activity.

Next, we examined the in vivo antitumor effect of the OVA/Al/high salt vaccine using the E.G7-OVA tumor model in mice. In either the prophylactic or therapeutic vaccine model, immunization of the OVA/Al/high salt complex inhibited tumor growth more effectively compared with the other groups (Figure $1 \mathrm{~K}$ ). The antitumor effect of the OVA/Al/3.6\% NaCl vaccine was further studied in an adoptive cellular/serum therapy model. Lymphocytes from mice immunized with OVA/Al/3.6\% $\mathrm{NaCl}$ effectively inhibited tumor growth, while the serum showed no apparent antitumor effect (Supplementary information, Figure S1M and S1N). To investigate the function of immune cell subsets in the antitumor activity elicited by the high salt formulation, we depleted $\mathrm{CD}^{+} /$ $\mathrm{CD} 8^{+} \mathrm{T}$ cells or NK cells through the injection of monoclonal antibodies. Only depletion of $\mathrm{CD}^{+} \mathrm{T}$ lymphocytes and NK cells showed effective abrogation of the antitumor effect induced by the OVA/Al/3.6\% NaCl vaccine (Supplementary information, Figure S1O). Taken together, these results suggest that a high-salt formulation of OVA/Al vaccine exhibited an enhanced antitumor effect in vivo through $\mathrm{CD} 8^{+} \mathrm{CTL}-m e d i a t e d$ cellular immunity independent of $\mathrm{CD} 4^{+} \mathrm{T}$ cells.

To further study the role of high-salt formulation in the strong antitumor effect, we assessed the changes in immune cell subsets after the immunization. Both central memory and effector memory $\mathrm{T}$ cells in $\mathrm{CD} 4^{+}$and $\mathrm{CD} 8^{+}$

Figure 1 Simultaneous enhancement of cellular and humoral immunity by the high-salt formulation of $\mathrm{Al}(\mathrm{OH})_{3}$ adjuvant. (A) High salt concentration enhances $\mathrm{Al}(\mathrm{OH})_{3}$ adjuvant-induced humoral immunity. C57BL/6 mice ( $n=5$ per group) were vaccinated subcutaneously three times with OVA/Al complex containing different concentrations of $\mathrm{NaCl}$ ( $5 \mu \mathrm{g}$ OVA per mouse). Seven days after the third immunization, the mouse serum was collected and levels of the total IgG and IgG subclasses and IgG1 titer were determined by ELISA. (B-E) OVA/Al/high salt vaccine induces specific cellular immunity. C57BL/6 mice were immunized in the same way as described in A. Lymphocytes were isolated from the spleen and further incubated in vitro with CD8 ${ }^{+}$-specific $O \mathrm{OVA}_{257-264}$ peptides (10 $\left.\mu \mathrm{g} / \mathrm{ml}\right)$ for 3 days. The generation of $\mathrm{CD}^{+}$CTLs was determined by FCM using PE-conjugated $\mathrm{H}-2 \mathrm{~K}^{\mathrm{b}} / \mathrm{OVA}_{257-264}$ tetramer (B). The expression of IFN- $\gamma$ was examined by FCM (C) and ELISA (D). The same lymphocytes were tested for CTL-mediated cytotoxicity against E.G7OVA cells by a standard $6 \mathrm{~h}{ }^{51} \mathrm{Cr}$ release assay (E). (F) High concentrations of $\mathrm{NaCl}$ promote the maturation of DCs in vitro. Bone marrow-derived DCs isolated from C57BL/6 mice were cultured in medium with the indicated $\mathrm{NaCl}$ concentrations for $48 \mathrm{~h}$ and the expression of maturation markers was analyzed by FCM. (G) High concentrations of $\mathrm{NaCl}$ promote the antigen uptake of DCs in vitro. DCs were incubated with $2 \mu \mathrm{g} / \mathrm{ml}$ Alexa Fluor 488-labeled OVA for $1 \mathrm{~h}$ at $37^{\circ} \mathrm{C}$ in medium with the indicated NaCl concentrations and analyzed under a fluorescent microscope. Scale bars, $20 \mu \mathrm{m}$. (H) High concentration of $\mathrm{NaCl}$ promotes secretion of the pro-inflammatory cytokines by DCs through the p38 MAPK pathway in vitro. DCs were pretreated with $10 \mu \mathrm{M} \mathrm{SB203580}$ (SB) or DMSO (DM) for $2 \mathrm{~h}$ and then treated with $0.9 \%$ or $1.5 \% \mathrm{NaCl}$ for $48 \mathrm{~h}$ in the presence of $5 \mu \mathrm{M} \mathrm{SB}$ or DM. The levels of pro-inflammatory cytokines in the supernatant were measured by ELISA. (I-J) High salt concentration induces antigen cross-presentation in DCs. DCs were cultured in medium with the indicated $\mathrm{NaCl}$ concentrations containing $10 \mu \mathrm{g} / \mathrm{ml} \mathrm{OVA}$. Then the stimulated DCs were co-cultured with CFSE-labeled CD8 ${ }^{+} \mathrm{T}$ cells from OT-I mice. The proliferation of T cells was assessed by FCM after 3 days of co-culture (I). DCs were treated with $2 \mu \mathrm{g} / \mathrm{ml}$ Alexa Fluor 488-labeled OVA in the presence of the indicated $\mathrm{NaCl}$ concentrations for $1 \mathrm{~h}$, stained with rabbit anti-LMP2/Cy3 antibody and DAPI, and visualized under a confocal laser scanning microscope. Scale bars, $5 \mu \mathrm{m}$ (J). (K) High-salt formulation potentiates the antitumor effect of OVA/AI vaccine in vivo. In a prophylactic model (left), C57BL/6 mice ( $n=10$ per group) were immunized with different vaccines for three times and then challenged subcutaneously with $3 \times 10^{6}$ E.G7-OVA cells 1 week after the third immunization. In a therapeutic model (right), mice ( $n=7$ per group) were treated by subcutaneous injection of different vaccines once a week for 3 weeks starting on day 3 after subcutaneously introduction of $3 \times 10^{6}$ E.G7-OVA cells. The tumor volume was measured every 3 days. (L) The high-salt formulation of OVA/AI diminished immune suppressive cells in tumor microenvironment. After the third treatment, a single-cell suspension of tumor tissues was prepared. The percentages of infiltrating CD $8^{+}$Iymphocytes, MDSCs and M2 macrophages were analyzed by FCM. 
T cell populations significantly increased in the OVA/ $\mathrm{Al} /$ high salt group (Supplementary information, Figure $\mathrm{S} 1 \mathrm{P})$. We also observed an increase in percentages of splenic $\mathrm{CD}^{+}$and $\mathrm{CD} 8^{+} \mathrm{T}$ cells in the OVA/Al/high salt group (Supplementary information, Figure S1P). As an immune suppressive tumor microenvironment might be a major determinant of the poor outcome of tumor immunotherapy [12], we further investigated whether the high-salt vaccine alters the immune microenvironment in tumors. We found that tumor-infiltrating lymphocytes increased, whereas myeloid-derived suppressor cells (MDSCs) and M2 macrophages decreased significantly in OVA/Al/3.6\% NaCl-treated mice (Figure 1L). These observations indicate that high salt-containing vaccine may alter the tumor microenvironment to favor more potent antitumor effects.

For safety assessment, no significant differences were observed between normal mice and the mice immunized with aluminum and high salt, as determined by appearance, body weight, fecal and urinary excretion, and H\&E staining of the vital organs. All the mice were under good physiological conditions as shown by the monitoring of heart rate and mean arterial pressure, even when the $\mathrm{NaCl}$ concentration reached as high as $14.4 \%$ (16 times higher than the normal osmolality; Supplementary information, Table S1).

In conclusion, we discovered a novel and safe formulation (high salt formulation) of $\mathrm{Al}(\mathrm{OH})_{3}$ adjuvant, which, aside from maintaining the induction of humoral immune response by normal Al-adjuvant, could further enhance the cellular immune response; and this effect may be closely related to the activation of and antigen cross-presentation by DCs. Consequently, the OVA/Al/ high salt formulation exhibited a significant antitumor effect against the E.G7-OVA tumor model in vivo, which might be largely due to $\mathrm{CD} 8^{+} \mathrm{CTL}-$-mediated cellular immunity and independent of $\mathrm{CD}^{+}{ }^{+} \mathrm{T}$ cells. The most interesting and surprising finding of this study is that the adjuvant effects could be significantly improved simply by altering the $\mathrm{NaCl}$ concentration in vaccines and such formulation is safe, easy to prepare, and of low cost. This concept may assist in the design of broad vaccine formulations and the development of safer and more effective adjuvants for therapeutic uses.

\section{Acknowledgments}

This work was supported by the National Key Research and Development Program of China (2016YFA0201402) and the National Natural Science Foundation of China (81602492).

Min Luo ${ }^{1, *}$, Bin Shao ${ }^{1, *}$, Jia-yun Yu ${ }^{1, *}$, Ting Liu ${ }^{1}$, Xiao Liang ${ }^{1}$, Lian Lu ${ }^{1}$, Ting-hong $\mathrm{Ye}^{1}$, Zhi-yao $\mathrm{He}^{1}$, Heng-yi Xiao ${ }^{1}$, Xia-wei Wei ${ }^{1}$

${ }^{1}$ Lab of Aging Research and Nanotoxicology, State Key Laboratory of Biotherapy, West China Hospital, Sichuan University, Chengdu, Sichuan 610041, China

*These three authors contributed equally to this work.

Correspondence: Xia-wei Wei

E-mail: xiaweiwei@scu.edu.cn

\section{References}

1 Reed SG, Orr MT, Fox CB. Nat Med 2013; 19:1597-1608.

2 McNutt M. Science 2013; 342:1417-1417.

3 Gupta RK. Adv DrugDel Rev 1998; 32:155-172.

4 Marrack P, McKee AS, Munks MW. Nat Rev Immunol 2009; 9:287293.

5 Steinman RM. Annu Rev Immunol 2012; 30:1-22.

6 Guermonprez P, Valladeau J, Zitvogel L, et al. Annu Rev Immunol 2002; 20:621-667.

7 Heath WR, Carbone FR. Annu Rev Immunol 2001; 19:47-64.

8 Kleinewietfeld M, Manzel A, Titze J, et al. Nature 2013; 496:518522.

9 Wu C, Yosef N, Thalhamer T, et al. Nature 2013; 496:513-517.

10 Zhang WC, Zheng XJ, Du LJ, et al. Cell Res 2015; 25:893-910.

11 Arthur JS, Ley SC. Nat Rev Immunol 2013; 13:679-692.

12 Kraman M, Bambrough PJ, Arnold JN, et al. Science 2010; 330:827830 .

(Supplementary information is linked to the online version of the paper on the Cell Research website.)

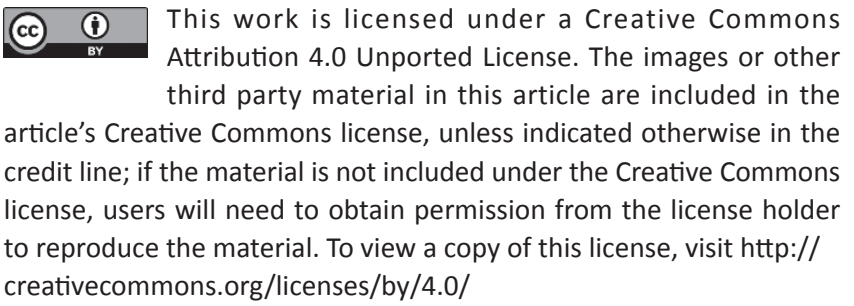

(c) The Author(s) 2017 